\title{
Domain walls and fermion scattering in Grand Unified models
}

\author{
D. A. Steel* \\ $A P C^{\dagger}, 11$ place Marcelin Berthelot, 75005 Paris, France \\ and \\ LPT, Université de Paris-Sud, \\ Bât. 210, 91405 Orsay Cedex, France.

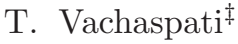 \\ CERCA, Department of Physics, Case Western Reserve University, \\ 10900 Euclid Avenue, Cleveland, OH 44106-7079, USA.
}

(Dated: October 4, 2018)

\begin{abstract}
Motivated by Grand Unification, we study the properties of domain walls formed in a model with $S U(5) \times Z_{2}$ symmetry which is spontaneously broken to $S U(3) \times S U(2) \times U(1) / Z_{6}$, and subsequently to $S U(3) \times U(1) / Z_{3}$. Even after the first stage of symmetry breaking, the $S U(3)$ symmetry is broken to $S U(2) \times U(1) / Z_{2}$ on the domain wall. In a certain range of parameters, flux tubes carrying colorand hyper-charge live on the domain wall and appear as "boojums" when viewed from one side of the domain wall. Magnetic monopoles are also formed in the symmetry breaking and those carrying color and hyper-charge can be repelled from the wall due to the Meissner effect, or else their magnetic flux can penetrate the domain wall in quantized units. After the second stage of symmetry breaking, fermions can transmute when they scatter with the domain wall, providing a simpler version of fermion-monopole scattering: for example, neutrinos can scatter into d-quarks, leaving behind electric charge and color which is carried by gauge field excitations living on the domain wall.
\end{abstract}

\section{INTRODUCTION}

The interactions of fermions with non-trivial scalar field profiles such as bubble walls, domain walls and other topological defects have been the subject of numerous studies (see 1, 2] for reviews). Depending on the model, a Yukawa-type coupling may lead to localized zero modes (and hence to the existence of current carrying cosmic strings for example), and it may also give non-trivial fermion scattering. Indeed, the calculation of the different reflection coefficients for particles and antiparticles scattering of electroweak bubble walls was central to the picture of electroweak baryogenesis put forward in the ' 80 s [1]. Though this picture is no longer valid, in [3] a similar mechanism was revived using embedded electroweak domain walls stabilized by thermal effects.

In this paper we study the interactions of bosons and fermions with domain walls in an $S U(5)$ model motivated by grand unification. Along the way we come across several novel phenomena which have not seen much discussion in the literature. We discover that magnetic monopoles carrying QCD color and hypercharge may interact non-trivially with the wall since $S U(3)$ color and $U(1)$ hypercharge are broken in the wall and vortices exist there. We also see that the scattering of fermions off the wall can lead to baryon and lepton number violation. This is not so surprising given that these numbers

${ }^{\dagger}$ UMR 7164 (CNRS, Université Paris 7, CEA, Observatoire de Paris)

*Electronic address: steer@apc.univ-paris7.fr

‡Electronic address: tanmay@monopole.phys.cwru.edu are not conserved in grand unified theories. However, the scattering can also deposit electric charge and $S U(3)$ charge on the domain wall when, for example, a neutrino is transmitted through the wall as a d-quark. Gauge field condensates carry this charge on the wall.

The domain walls are formed in the first stage of the symmetry breaking scheme

$$
\begin{gathered}
S U(5) \times Z_{2} \quad \stackrel{\boldsymbol{H}}{\longrightarrow} \frac{S U(3) \times S U(2) \times U(1)}{Z_{6}} \\
\stackrel{\vec{\phi}}{\longrightarrow} \frac{S U(3) \times U(1)}{Z_{3}}
\end{gathered}
$$

where $\boldsymbol{H}$ is a 24 component $S U(5)$ adjoint Higgs while $\vec{\phi}$ is a 5-component complex Higgs transforming in the fundamental representation of $S U(5)$. The vacuum manifold for the first symmetry breaking is disconnected due to the $Z_{2}$ factor; for the second stage it is connected and no further topological defects are formed. (Monopoles are also formed in the first stage of symmetry breaking.) Our motivation for studying this problem is three fold. Firstly, the non-abelian domain walls produced in the first symmetry breaking have many unusual properties compared to standard $Z_{2}$ domain walls. As discussed in Refs. [4, 5, 6, 7], in fact three distinct classes of domain walls are formed in this phase transition (see also Refs. 8, 9, 10] for a discussion of the resulting lattice of domain walls). Only one of these walls is stable and the full $S U(5) \times Z_{2}$ symmetry is not restored in its core. On the contrary, the symmetry inside the stable wall is smaller than the unbroken $S U(3) \times S U(2) \times U(1)$ symmetry. A second motivation is to explore grand unified phase transitions. Our analysis shows that many complex and diverse outcomes are likely, and the eventual 
cosmological consequences of a grand unified phase transition depend on the structure and interactions of both stable and unstable topological defects in the model.

A final motivation is to understand how fermions and monopoles interact with such a wall. Here we work in the context of $S U(5)$ grand unified theory (GUT), even though this model is ruled out for a number of reasons including proton decay. However, other (supersymmetric) GUTs often break down to the standard model through $S U(5) \times Z_{2}$ and hence phenomena similar to those discussed here may also occur in more realistic GUTs. In this context, $\boldsymbol{H}$ breaks the first symmetry in Eq. (1) at scales

$$
v_{\mathrm{GUT}} \simeq 10^{16} \mathrm{GeV}
$$

whereas fermions get their mass from Yukawa interactions with the electroweak Higgs $\vec{\phi}$ at scales

$$
v_{\mathrm{EW}} \simeq 100 \mathrm{GeV} \text {. }
$$

Given this hierarchy one can neglect the backreaction of the fermions and $\vec{\phi}$ on the GUT domain walls. However, due to the standard couplings between $\boldsymbol{H}$ and $\vec{\phi}$ (see, for instance [11, 12]), after electroweak symmetry breaking, GUT domain walls will lead to regions in space in which the electroweak Higgs take different values. As mentioned above, the result is that as fermions go from one region to another they can scatter in non-trivial ways.

Before studying this problem, it will be useful to recall the main features of fermion scattering off the familiar $Z_{2}$ domain wall, and at the same time introduce some relevant notation. The action for this coupled system is $S(\Psi, \phi)=S_{1}(\phi)+S_{2}(\Psi, \phi)$ where $\phi$ is a real scalar field, $\Psi$ a Dirac fermion, and

$$
\begin{aligned}
S_{1}(\phi) & =\int d^{4} x\left(\frac{1}{2}\left(\partial_{\mu} \phi\right)^{2}-\frac{\lambda}{4}\left(\phi^{2}-\eta^{2}\right)^{2}\right) \\
S_{2}(\Psi, \phi) & =\int d^{4} x(\bar{\Psi}(i \not \partial+g \phi) \Psi) \\
& =\int d^{4} x\left(i\left(\bar{\Psi}_{L} \not \partial \Psi_{L}+\bar{\Psi}_{L}^{C} \not \partial \Psi_{L}^{C}\right)\right. \\
& \left.+g \phi\left[\Psi_{L}^{\mathrm{T}} C \Psi_{L}^{C}-\left(\Psi_{L}^{C}\right)^{\dagger} C\left(\Psi_{L}\right)^{*}\right]\right) .
\end{aligned}
$$

In Eq. (4) we have used the standard notation for GUTs and decomposed $\Psi$ into left- and right- handed components, introducing the charge conjugate field

$$
\Psi=\Psi_{L}+\Psi_{R}=\Psi_{L}+i \gamma^{2}\left(\psi_{L}^{C}\right)^{*}
$$

where $\Psi_{L, R}=\left(1 \mp \gamma^{5}\right) \Psi / 2$ and

$$
\Psi_{L}^{C} \equiv\left(\Psi^{C}\right)_{L}, \quad \Psi^{C}=C \gamma^{0} \Psi, \quad C=i \gamma^{2} \gamma^{0} .
$$

The $\gamma$ matrices satisfy $\left\{\gamma^{\mu}, \gamma^{\nu}\right\}=2 \eta^{\mu \nu},\left(\gamma^{\mu}\right)^{\dagger}=\gamma^{0} \gamma^{\mu} \gamma^{0}$, and $\gamma^{5} \equiv i \gamma_{0} \gamma_{1} \gamma_{2} \gamma_{3}$. We work in the Dirac representation;

$$
\gamma^{0}=\left(\begin{array}{cc}
1 & 0 \\
0 & -1
\end{array}\right), \gamma^{i}=\left(\begin{array}{cc}
0 & \sigma^{i} \\
-\sigma^{i} & 0
\end{array}\right), \gamma^{5}=\left(\begin{array}{ll}
0 & 1 \\
1 & 0
\end{array}\right)
$$

From Eqs. (3) and (4), the fermion equation of motion is

$$
i \not \partial \Psi+g \phi \Psi=0
$$

or equivalently

$$
i \not \partial \Psi_{L}-g \phi \gamma^{0} C\left(\Psi_{L}^{C}\right)^{*}=0, \quad i \not \partial \Psi_{L}^{C}-g \phi \gamma^{0} C \Psi_{L}^{*}=0 .
$$

If backreaction is ignored, the domain wall solution centered on $y=0$ and interpolating between the two discrete vacua at $\phi= \pm \eta$ is given by the solution

$$
\phi_{D W}(y)=\eta \tanh (\tilde{\sigma} y)
$$

where $\tilde{\sigma}=\lambda \eta^{2} / \sqrt{2}$. At the center of the wall $\phi_{D W}=0$ and the $Z_{2}$ symmetry is restored. There exists a fermion zero mode that is localized on the wall [2]. Similar comments will not hold for $S U(5) \times Z_{2}$ domain walls. Fermions may also scatter off the wall, in which case the asymptotic states for Eq. (8) consist of incoming and reflected positive energy (spin up, say) plane waves at $y=-\infty$

$$
\Psi_{I}=e^{-i(\omega t-k y)} u_{\uparrow}(k, m), \Psi_{R}=\alpha e^{-i(\omega t+k y)} u_{\uparrow}(-k, m)
$$

where $m=g \eta$, and a transmitted wave at $y=+\infty$

$$
\Psi_{T}=\beta e^{-i(\omega t-k y)} u_{\uparrow}(k,-m) .
$$

Here $\alpha$ and $\beta$ are $c$-numbers, and $\omega^{2}-k^{2}=m^{2}$. The normalized spinor $u_{\uparrow}(k, m)$ satisfies $(\not k-m) u_{\uparrow}(k, m)=0$ so that $u_{\uparrow}(k, m)^{\mathrm{T}}=\sqrt{\omega+m}(1,0,0, i k /(\omega+m))$. By squaring Eq. (8) and rewriting in terms of the variable $2 z=1-\tanh (\tilde{\sigma} y)$, one obtains a hypergeometric equation that can be solved exactly. The result may be found in [13, 14], and one can calculate for example, the reflection and transmission coefficients

$$
R=\left|\frac{j_{R}^{(2)}}{j_{I}^{(2)}}\right|, T=\left|\frac{j_{T}^{(2)}}{j_{I}^{(2)}}\right|
$$

where the current $j^{(\mu)}$ is defined as the expectation value of the normal-ordered quantum operator : $\bar{\Psi} \gamma^{\mu} \Psi$ :. In the limit of a zero thickness wall, Eq. (10) reduces to

$$
\phi_{D W}(y)=2 \eta\left[\Theta(y)-\frac{1}{2}\right] \text {, }
$$

and $R$ and $T$ are straightforward to calculate. The solution of the Dirac equation, Eq. (8), is obtained by matching the plane waves across the wall, $u_{\uparrow}(k, m)+$ $\alpha u_{\uparrow}(-k, m)=\beta u_{\uparrow}(k,-m)$, leading to

$$
R=\frac{m^{2}}{\omega^{2}}, \quad T=\frac{\omega^{2}-m^{2}}{\omega^{2}} .
$$

In the remainder of this paper we tackle a similar problem for $S U(5) \times Z_{2}$ domain walls. In Sec. I we discuss the stable domain wall solutions in the $S U(5) \times Z_{2}$ model, formed in the first stage of symmetry breaking (Eq. (1)). In Sec. III we describe the effects of electroweak symmetry breaking on the domain wall, and how this can lead, for example, to chromomagnetic vortices on the wall. Fermion scattering is discussed in Sec. IV] 


\section{DOMAIN WALLS IN $S U(5) \times Z_{2}$}

In this section we briefly recall the main results of $[4,5$, 6. regarding the properties of domain walls formed in the first symmetry breaking Eq. (11). Electroweak symmetry breaking will be considered in the following section. The Langrangian density is*

$$
\mathcal{L}_{\boldsymbol{H}}=\operatorname{Tr}\left[\left(\boldsymbol{D}_{\mu} \boldsymbol{H}\right)\left(\boldsymbol{D}^{\mu} \boldsymbol{H}\right)^{\dagger}\right]-V(\boldsymbol{H})+\frac{1}{2} \operatorname{Tr}\left[F_{\mu \nu} F^{\mu \nu}\right]
$$

where $\boldsymbol{D}_{\mu} \boldsymbol{H}=\partial_{\mu} \boldsymbol{H}+i e\left[\boldsymbol{A}_{\mu}, \boldsymbol{H}\right]$ and $F_{\mu \nu}=\partial_{\mu} \boldsymbol{A}_{\nu}-$ $\partial_{\nu} \boldsymbol{A}_{\mu}+i e\left[\boldsymbol{A}_{\mu}, \boldsymbol{A}_{\nu}\right]$. The potential $V(\boldsymbol{H})$ is taken to be quartic in the adjoint Higgs $\boldsymbol{H}$;

$$
V(\boldsymbol{H})=-m_{1}^{2} \operatorname{tr}\left(\boldsymbol{H}^{2}\right)+\lambda_{1}\left(\operatorname{tr} \boldsymbol{H}^{2}\right)^{2}+\lambda_{2} \operatorname{tr}\left(\boldsymbol{H}^{4}\right)+V_{0}
$$

with $V_{0}$ chosen such that at its minimum, $V=0$. Action (16) is invariant under local $S U(5)$ transformations

$$
\begin{aligned}
& \boldsymbol{H} \stackrel{S U(5)}{\longrightarrow} \boldsymbol{H}^{\prime}=\boldsymbol{g} \boldsymbol{H} \boldsymbol{g}^{\dagger} \\
&\left(\boldsymbol{D}_{\mu} \boldsymbol{H}\right) \stackrel{S U(5)}{\longrightarrow}\left(\boldsymbol{D}_{\mu} \boldsymbol{H}\right)^{\prime}=\boldsymbol{g}\left(\boldsymbol{D}_{\mu} \boldsymbol{H}\right) \boldsymbol{g}^{\dagger} \\
& \boldsymbol{A}_{\mu} \stackrel{S U(5)}{\longrightarrow} \boldsymbol{A}_{\mu}^{\prime}=\boldsymbol{g} \boldsymbol{A}_{\mu} \boldsymbol{g}^{\dagger}-\frac{i}{e}\left(\partial_{\mu} \boldsymbol{g}\right) \boldsymbol{g}^{\dagger}
\end{aligned}
$$

as well as $Z_{2}$ transformations

$$
\boldsymbol{H} \stackrel{Z_{2}}{\longrightarrow} \boldsymbol{H}^{\prime \prime}=-\boldsymbol{H}
$$

which leave the gauge sector invariant. Notice that this $Z_{2}$ transformation is not included in the $S U(5)$ transformation: for any $g \in S U(5)$ one has $\operatorname{Tr}\left(\boldsymbol{H}^{\prime 3}\right)=$ $\operatorname{Tr}\left(\boldsymbol{g} \boldsymbol{H}^{3} \boldsymbol{g}^{\dagger}\right)=\operatorname{Tr}\left(\boldsymbol{H}^{3}\right)$, whilst under $Z_{2} \operatorname{Tr}\left(\boldsymbol{H}^{3}\right)$ changes sign. Thus the action Eq. (16) is $S U(5) \times Z_{2}$ invariant provided there are no cubic terms in the potential. ${ }^{\dagger}$

As discussed in e.g. 11, 12], the choice of parameters

$$
\lambda_{2} \geq 0, \quad \frac{\lambda_{1}}{\lambda_{2}} \geq-\frac{7}{30}, \quad m_{1}^{2}>0
$$

breaks $S U(5) \times Z_{2} \longrightarrow S U(3) \times S U(2) \times U(1) / Z_{6}$. Following [6], the domain wall solution is known analytically if

$$
\lambda_{1}=-3 \lambda_{2} / 20
$$

The potential is minimized for

$$
\boldsymbol{H}_{0}=v_{G U T} \operatorname{diag}(2,2,2,-3,-3)
$$

\footnotetext{
* Our notation is the following. The $a=1, \ldots, 24$ hermitian and traceless generators of $S U(5)$ are denoted by $\boldsymbol{T}_{a}$, and they are normalized such that $\operatorname{Tr}\left(\boldsymbol{T}_{a} \boldsymbol{T}_{b}\right)=\delta_{a b} / 2$. We often write $\boldsymbol{H}=$ $H^{a} \boldsymbol{T}_{a}$. A group element of $S U(5)$ is denoted by $\boldsymbol{g}=\exp \left(i \epsilon_{a} \boldsymbol{T}_{a}\right)$ with $\operatorname{det} \boldsymbol{g}=1$ and $\boldsymbol{g g}^{\dagger}=\mathbf{1}$.

$\dagger$ One should note a subtlety regarding this $Z_{2}$ transformation. There are certain field configurations, $\boldsymbol{H}_{s}$, satisfying $\operatorname{Tr}\left(\boldsymbol{H}_{s}^{2 n+1}\right)=0$ with $n \in \mathcal{Z}$ for which one can change the sign of $\boldsymbol{H}_{s}$ using an $S U(5)$ transformation. An example is $\overline{\boldsymbol{H}}_{s} \equiv$ $\operatorname{diag}(1,-1,0,0,0)$ : for $\boldsymbol{g}=\left(\sigma_{1}, 1,1,-1\right), \overline{\boldsymbol{H}}_{s}^{\prime}=\boldsymbol{g} \overline{\boldsymbol{H}}_{s} \boldsymbol{g}^{\dagger}=-\overline{\boldsymbol{H}}_{s}$.
}

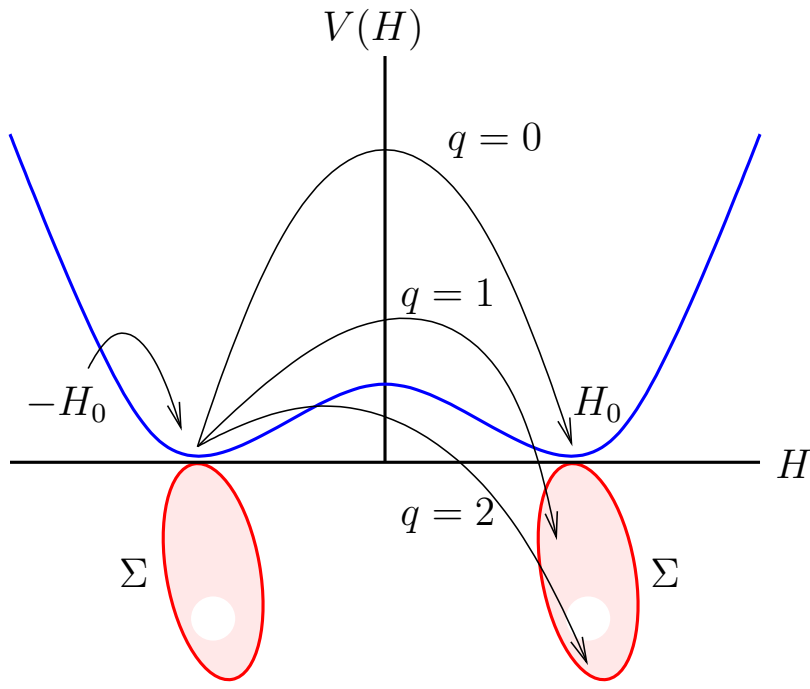

FIG. 1: The vacuum manifold for $S U(5) \times Z_{2}$ breaking is in two identical disconnected pieces, each labeled by $\Sigma$. $\Sigma$ itself has non-trivial topology, including holes which lead to $\Pi_{2}(\Sigma) \neq 0$ and hence monopoles. Different domain wall solutions, labeled by $q=0,1,2$, are obtained for different boundary conditions at spatial infinity.

where

$$
v_{G U T}^{2}=\frac{m_{1}^{2}}{5 \lambda_{2}},
$$

and $\boldsymbol{H}_{0}$ is invariant under the unbroken subgroup

$$
K_{0}=S U(3) \times S U(2) \times U(1) / Z_{6}
$$

so that

$$
\boldsymbol{H}_{0}=\mathbf{h} \boldsymbol{H}_{0} \boldsymbol{h}^{\dagger} \quad \forall \mathbf{h} \in K_{0} .
$$

It is straightforward to identify the $(8+3+1)=12$ generators of $K_{0}$ out of the 24 generators of $S U(5)$ (see e.g. 11]).

The vacuum manifold of the potential given in Eq. (17) has two discrete sectors linked by the $Z_{2}$ transformation. Furthermore, each sector consists of a whole manifold of vacua generated by $S U(5)$ transformations (see Fig. 11 for a schematic representation). This should be contrasted with the standard potential of Eq. (2). Correspondingly, the properties of the resulting domain walls are more diverse.

By symmetry, the only non-trivial component of the gauge field in the static domain wall will be $\boldsymbol{A}^{y}$. This can always be set to zero by a gauge transformation and hence in the following discussion we suppose that all gauge fields vanish.

Suppose one fixes

$$
\boldsymbol{H}(y \rightarrow-\infty)=\boldsymbol{H}_{0} .
$$

Then topologically non-trivial boundary conditions occur for $\boldsymbol{H}(y \rightarrow \infty) \equiv \boldsymbol{H}^{+}$given by

$$
\boldsymbol{H}^{+}=-\boldsymbol{g H}_{0} \boldsymbol{g}^{\dagger} \quad \forall \boldsymbol{g} \in S U(5) .
$$


Suppose $\boldsymbol{g} \in K_{0}$. Then $\boldsymbol{H}^{+}=-\boldsymbol{H}_{0}$ (see Fig. 10, but the resulting " $q=0$ " domain wall is unstable $[4,5,6]$. The only stable domain wall lies in the " $q=2$ " class [4, 5, 6] for which the boundary condition at $+\infty$ is

$$
\boldsymbol{H}_{(q=2)}^{+}=-v_{\mathrm{GUT}} \operatorname{diag}(2,-3,-3,2,2) .
$$

The explicit domain wall solution can be written in closed form for the choice of parameters in Eq. (23):

$$
\begin{aligned}
& \boldsymbol{H}_{(q=2)}(y)=\frac{v_{G U T}}{2}[\tanh (\sigma y) \operatorname{diag}(-4,1,1,1,1) \\
& \quad+\operatorname{diag}(0,5,5,-5,-5)] \\
& \boldsymbol{A}_{(q=2)}^{\mu}(y)=0 .
\end{aligned}
$$

Observe that the full $S U(5)$ symmetry is not restored at the center of the wall since the unbroken symmetry there is $S U(2)^{2} \times U(1)^{2}$, which is smaller than the unbroken $S U(3) \times S U(2) \times U(1)$. In the wall color symmetry is broken: $S U(3) \rightarrow S U(2) \times U(1) / Z_{3}$.

We now focus on these " $q=2$ " walls and determine their effect on the electroweak Higgs $\vec{\phi}$ and hence on electroweak symmetry breaking.

\section{EFFECT OF ELECTROWEAK SYMMETRY BREAKING ON THE DOMAIN WALL}

So far we have considered the GUT symmetry breaking which is the first part of Eq. (1). Now we turn to the second part of Eq. (10) in which the electroweak symmetry breaks due to the VEV of the scalar field, $\vec{\phi}$. In the next two subsections, we will first discuss the bosonic sector, focusing on the solution for $\vec{\phi}$ in the background of the " $q=2$ " GUT domain wall. Then we discuss the fermionic sector in the background of the domain wall which now includes the solution for $\vec{\phi}$. Scattering of fermions off the domain wall is considered section IV

\section{A. Bosonic sector}

The Lagrangian density for the bosonic sector of the GUT theory is [1]

$$
\mathcal{L}=\mathcal{L}_{\boldsymbol{H}}+\left(\boldsymbol{D}_{\mu} \vec{\phi}\right)\left(\boldsymbol{D}^{\mu} \vec{\phi}\right)^{\dagger}-V(\vec{\phi})-V(\boldsymbol{H}, \phi)
$$

where $\mathcal{L}_{\boldsymbol{H}}$ is given in Eq. (16), and

$$
\begin{aligned}
V(\vec{\phi}) & =-m_{2}^{2}\left(\vec{\phi}^{\dagger} \vec{\phi}\right)+\lambda_{3}\left(\vec{\phi}^{\dagger} \vec{\phi}\right)^{2} \\
V(\boldsymbol{H}, \vec{\phi}) & =\lambda_{4}\left(\operatorname{tr} \boldsymbol{H}^{2}\right)\left(\vec{\phi}^{\dagger} \vec{\phi}\right)+\lambda_{5}\left(\vec{\phi}^{\dagger} \boldsymbol{H}^{2} \vec{\phi}\right)
\end{aligned}
$$

with $m_{2}^{2}>0$ and $\lambda_{3}>0$. Under $S U(5)$ and $Z_{2}$

$$
\vec{\phi} \stackrel{S U(5)}{\longrightarrow} \vec{\phi}^{\prime}=\boldsymbol{g} \vec{\phi}, \quad \vec{\phi} \stackrel{Z_{2}}{\longrightarrow} \vec{\phi}^{\prime \prime}=+\vec{\phi}
$$

Note that the Lagrangian is also invariant under the transformation $(\boldsymbol{H}, \vec{\phi}) \rightarrow(\boldsymbol{H},-\vec{\phi})$ for any $(\boldsymbol{H}, \vec{\phi})$. This is an additional $Z_{2}^{\prime}$ symmetry of the model. However, the $Z_{2}^{\prime}$ symmetry, in combination with $S U(5)$ rotations, remains unbroken throughout, and we shall ignore it in the remainder of this paper.

Given the domain wall solution of Eq. (31), one can determine $\vec{\phi}$ in this background. From Eqs. (34) and (35), the effective potential for $\vec{\phi}$ is

$$
\begin{aligned}
\tilde{V}(|\vec{\phi}|) & =V(\vec{\phi})+V(\boldsymbol{H}, \vec{\phi}) \\
& =\left|\phi_{1}\right|^{2} \mu_{1}^{2}(y)+\left(\left|\phi_{2}\right|^{2}+\left|\phi_{3}\right|^{2}\right) \mu_{23}^{2}(y) \\
& +\left(\left|\phi_{4}\right|^{2}+\left|\phi_{5}\right|^{2}\right) \mu_{45}^{2}(y)+\lambda_{3} \sum_{p=1}^{5} \sum_{q \neq p}\left|\phi_{p}\right|^{2}\left|\phi_{q}\right|^{2} \\
& +\lambda_{3} \sum_{p=1}^{5}\left|\phi_{p}\right|^{4}
\end{aligned}
$$

where $p=1, \ldots, 5$ labels the components of $\vec{\phi}$ and

$$
\begin{aligned}
\mu_{1}^{2}(y) & \left.=-m_{2}^{2}+v_{\mathrm{GUT}}^{2}\left[25 \lambda_{4}+\left(5 \lambda_{4}+4 \lambda_{5}\right) \tanh ^{2}(\sigma y)\right] 38\right) \\
\mu_{23}^{2}(y) & =-m_{2}^{2}+v_{\mathrm{GUT}}^{2}\left[25\left(\lambda_{4}+\frac{\lambda_{5}}{4}\right)\right. \\
& \left.+5\left(\lambda_{4}+\frac{\lambda_{5}}{20}\right) \tanh ^{2}(\sigma y)+\frac{5 \lambda_{5}}{2} \tanh (\sigma y)\right] \\
\mu_{45}^{2}(y) & =\mu_{23}^{2}(-y) .
\end{aligned}
$$

Asymptotically

$$
\begin{aligned}
\mu_{1}^{2}( \pm \infty) & =\mu_{23}^{2}(-\infty)=\mu_{45}^{2}(+\infty) \\
& =-m_{2}^{2}+v_{\mathrm{GUT}}^{2}\left[30 \lambda_{4}+4 \lambda_{5}\right] \equiv \mu_{S U(3)}^{2} \\
\mu_{23}^{2}(+\infty) & =\mu_{45}^{2}(-\infty) \\
& =-m_{2}^{2}+v_{\mathrm{GUT}}^{2}\left[30 \lambda_{4}+9 \lambda_{5}\right] \equiv \mu_{S U(2)}^{2}
\end{aligned}
$$

and from Eqs. (24) and (30) the boundary conditions are

$$
\vec{\phi}(y \rightarrow-\infty)=\left(\begin{array}{c}
0 \\
0 \\
0 \\
0 \\
v_{\mathrm{EW}}
\end{array}\right), \quad \vec{\phi}(y \rightarrow+\infty)=\left(\begin{array}{c}
0 \\
0 \\
v_{\mathrm{EW}} \\
0 \\
0
\end{array}\right)
$$

with associated unbroken $\mathrm{U}(1)$ generators

$$
\begin{aligned}
& Q(y \rightarrow-\infty)=\operatorname{diag}(-1 / 3,-1 / 3,-1 / 3,1,0) \\
& Q(y \rightarrow+\infty)=\operatorname{diag}(-1 / 3,1,0,-1 / 3,-1 / 3)
\end{aligned}
$$

These boundary conditions are obtained from the potential in Eq. (37) provided

$$
\mu_{S U(3)}^{2}+2 \lambda_{3} v_{\mathrm{EW}}^{2}>0, \quad \mu_{S U(2)}^{2}<0
$$

so that

$$
v_{\mathrm{EW}}^{2}=\frac{\left|\mu_{S U(2)}^{2}\right|}{2 \lambda_{3}}=-\frac{\mu_{S U(2)}^{2}}{2 \lambda_{3}} .
$$


Furthermore, from Eq. (46) $\mu_{S U(3)}^{2}-\mu_{S U(2)}^{2}>0$ and hence Eqs. (41) and (42) imply that $\lambda_{5}<0$. If one assumes that there are no fine tunings between the coupling constants, the hierarchy $v_{\text {EW }}^{2} / v_{\text {GUT }}^{2} \equiv \epsilon \simeq 10^{-24}$ implies that [1, 12],

$$
\lambda_{a} \equiv \lambda_{4}+\frac{3}{10} \lambda_{5}>0
$$

so that $\lambda_{4}>0$. Finally, it will be useful to define

$$
r=-\frac{\lambda_{5}}{\lambda_{a}}
$$

In a realistic scenario the different coupling constants are temperature dependent and the constraints apply to the effective coupling constants.

\section{1. $\phi_{1}$ condensate and monopole interactions}

Given these signs of coupling constants, there may be a condensate of $\phi_{1}$ on the wall (both before and after the electroweak phase transition). Indeed, from Eq. (38), $\mu_{1}^{2}(0)<0$ provided $r<2 / 5$. This condensate transforms non-trivially under $S U(3)$ color and $U(1)$ hypercharge symmetries, and hence leads to non-trivial interactions with any magnetic monopoles also carrying the same charges. Let us denote the relevant magnetic flux by $\mathbf{B}^{\prime}$ (see Fig. 21). If the $\phi_{1}$ condensate is uniform, the monopole can be repelled from the wall through the Meissner effect. The force can be found using the method of images and is inversely proportional to the square of the distance between the monopole and the wall. However, there is also another possibility: since $\phi_{1}$ is a complex field, and since the condensate is localized on the wall, there could be vortices situated on the wall due to the winding of $\phi_{1}$. (This is the analogue of a "boojum" in condensed matter physics — that is, a defect that can only live on the surface of a container because of the boundary conditions.) Now, depending on the relative orientation of the condensate and the magnetic monopole charge, the magnetic field due to the monopole could pass through the wall in quantized units of flux in the form of a vortex.

Another intriguing point linked to this condensate is that it implies, at least classically, the existence of massive fermions on the wall, since the fermions get their mass from interactions with the electroweak Higgs (see Sec. 【IB). Thus, before the electroweak phase transition it would seem to be possible to have massless fermions off the wall but massive fermions confined on it.

We hope to return to a more detailed investigation of both these possibilities in the future.

\section{No $\phi_{1}$ condensate}

In the remainder of this paper we focus on the case in which $r>2 / 5$ so that $\phi_{1}(y)=0$. The fields $\phi_{2}$ and
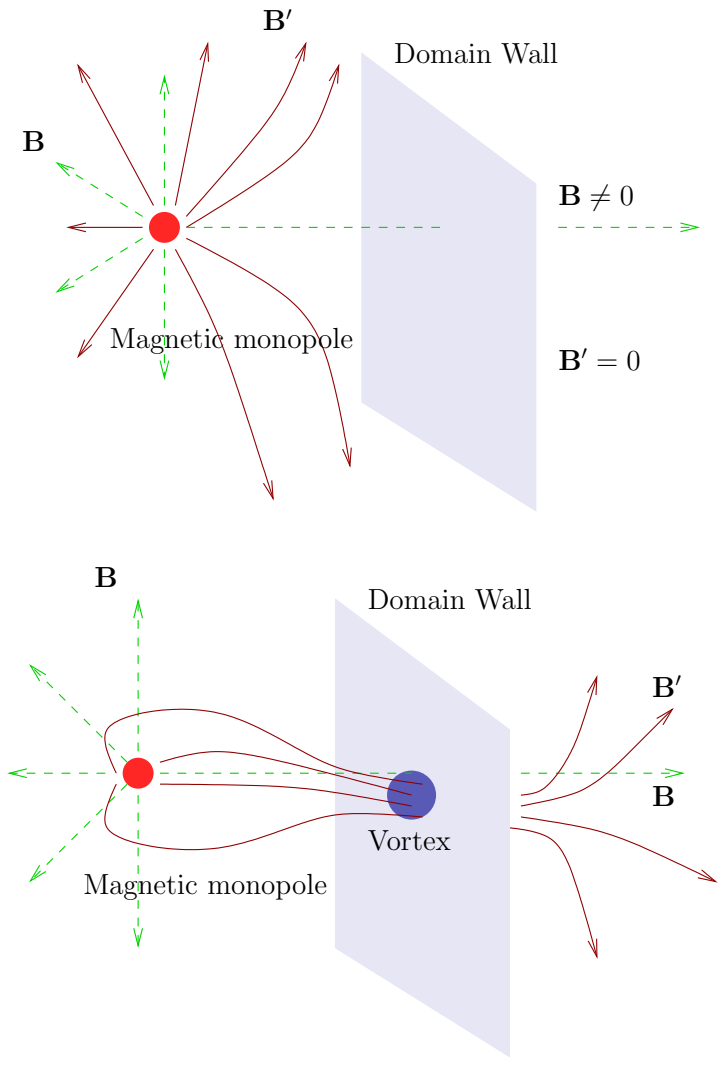

FIG. 2: On the wall a combination of the $S U(3)$ and hypercharge magnetic fields (denoted by $\mathbf{B}^{\prime}$ ) is massive due to the $\phi_{1}$ condensate. A magnetic monopole sourcing $\mathbf{B}^{\prime}$ is repelled from the wall due to the Meissner effect, as shown in the top figure. Alternatively, the $\mathbf{B}^{\prime}$ magnetic flux passes through the wall in a flux tube and the monopole is attracted to the domain wall, eventually being pulled through the wall (second figure). The orthogonal components of the monopole magnetic field, denoted by $\mathbf{B}$, do not interact with the wall.

$\phi_{4}$ can be set to zero so that the remaining degrees of freedom are $\phi_{3,5}$, and thus the profile of $\vec{\phi}$ is

$$
\vec{\phi}(y)=\left(\begin{array}{c}
0 \\
0 \\
\alpha(y) \\
0 \\
\beta(y)
\end{array}\right) \equiv \sqrt{\alpha^{2}+\beta^{2}}\left(\begin{array}{c}
0 \\
0 \\
\cos \theta \\
0 \\
\sin \theta
\end{array}\right)
$$

where the asymptotic behavior of $\alpha(y)$ and $\beta(y)$ is fixed by Eq.431), and

$$
\tan \theta=\frac{\beta(y)}{\alpha(y)}
$$

so that

$$
\theta(-\infty)=\frac{\pi}{2}, \quad \theta(+\infty)=0 .
$$

For the following analysis it will be convenient to change gauge and work in a gauge in which $\vec{\phi}(y)$ is nonzero only in the 5 th entry. Consider therefore $\boldsymbol{g}(y) \in$ 
$S U(5)$ given by

$$
\boldsymbol{g}(y)=\left(\begin{array}{ccccc}
1 & 0 & 0 & 0 & 0 \\
0 & \sin \theta & 0 & -\cos \theta & 0 \\
0 & 0 & \sin \theta & 0 & -\cos \theta \\
0 & \cos \theta & 0 & \sin \theta & 0 \\
0 & 0 & \cos \theta & 0 & \sin \theta
\end{array}\right)
$$

with $\boldsymbol{g}(-\infty)=\operatorname{diag}(1,1,1,1,1)$. Then after a gauge transformation $(\vec{\phi}(y) \rightarrow \boldsymbol{g}(y) \vec{\phi}(y))$,

$$
\vec{\phi}(y)=\sqrt{\alpha(y)^{2}+\beta(y)^{2}}\left(\begin{array}{l}
0 \\
0 \\
0 \\
0 \\
1
\end{array}\right),
$$

and

$$
\boldsymbol{A}_{y}=\frac{i}{e} \frac{d \theta}{d y}\left(\begin{array}{ccccc}
0 & 0 & 0 & 0 & 0 \\
0 & 0 & 0 & 1 & 0 \\
0 & 0 & 0 & 0 & 1 \\
0 & -1 & 0 & 0 & 0 \\
0 & 0 & -1 & 0 & 0
\end{array}\right)
$$

The gauge transformation (Eq. (18) ) of $\boldsymbol{H}$ yields

$$
\boldsymbol{H}(-\infty)=\boldsymbol{H}_{0}=-\boldsymbol{H}(+\infty)
$$

and finally, in this gauge, $Q(-\infty)=Q(+\infty)$ as given by Eq. (44). Note that in the thin wall approximation, which we shall be using later,

$$
\alpha(y)=v_{\mathrm{EW}} \Theta(y), \beta(y)=v_{\mathrm{EW}} \Theta(-y),
$$

and

$$
\frac{d \theta}{d y} \simeq-\frac{\pi \delta(y)}{2} .
$$

Furthermore, in this limit where $\boldsymbol{H}(y) \propto \boldsymbol{H}_{0}$, one can see from Eq. (44) that $\boldsymbol{A}_{y}$ carries electromagnetic charge since $\left[Q, \boldsymbol{A}_{y}\right] \neq 0$. Similarly, it can also carry color charge (indeed $\boldsymbol{A}_{y}$ is a lepto-quark boson [12]). Finally, we note that the $\boldsymbol{g}(y)$ of Eq. (53) is not unique: there exist many other gauge transformations for which Eqs. (54) and (56) are also satisfied. Some of these transformations may have the advantage of yielding gauge fields with definite charges. Despite that we use the simpler transformation Eq. (53), and now turn to the fermion equations of motion.

\section{B. Fermions}

The Lagrangian for the fermionic sector is 11,12

$$
\begin{aligned}
\mathcal{L}_{f} & =i\left(\bar{\psi}_{p}\right)_{L} \not D\left(\psi_{p}\right)_{L}+i \bar{\chi}_{L}^{p q} \not \supset \chi_{L}^{p q} \\
& +g_{1}\left[\left(\psi_{p}\right)_{L}^{\mathrm{T}} C \chi_{L}^{p q} \phi_{q}^{\dagger}-\phi_{q} \bar{\chi}_{L}^{p q} \gamma^{0} C\left(\psi_{p}\right)_{L}^{*}\right] \\
& +g_{2} \epsilon_{p q r s t}\left[\left(\chi_{L}^{p q}\right)^{\mathrm{T}} C \chi_{L}^{r s} \phi^{t}-\left(\phi^{t}\right)^{\dagger} \bar{\chi}_{L}^{p q} \gamma^{0} C\left(\chi_{L}^{r s}\right)^{*}\right]
\end{aligned}
$$

where $\psi$ is a vector transforming as $\overline{5}$, and $\chi$ is an antisymmetric tensor 10 , so that the Lagrangian is invariant under $\vec{\psi} \rightarrow \boldsymbol{g}^{*} \vec{\psi}, \chi \rightarrow \boldsymbol{g} \chi \boldsymbol{g}^{\mathrm{T}}$ and $\vec{\phi} \rightarrow \boldsymbol{g} \vec{\phi}$. The equations of motion are

$$
\begin{gathered}
i\left[\not \partial\left(\psi_{p}\right)_{L}-i e \gamma^{2}\left(\boldsymbol{A}_{2}^{*}\right)_{p q}\left(\psi_{q}\right)_{L}\right]-g_{1} \gamma^{0} C\left(\chi_{L}^{p q}\right)^{*} \phi_{q}=0(60) \\
i\left[\not \partial \chi_{L}^{p q}+2 i e \gamma^{2}\left(\boldsymbol{A}_{2}\right)_{p r} \chi_{L}^{r q}\right]+\frac{g_{1}}{2} \gamma^{0} C\left[\phi_{p}\left(\psi_{q}\right)_{L}^{*}-\phi_{q}\left(\psi_{p}\right)_{L}^{*}\right] \\
-2 g_{2} \epsilon_{r s p q t}\left(\phi^{t}\right)^{\dagger} \gamma^{0} C\left(\chi_{L}^{r s}\right)^{*}=0
\end{gathered}
$$

The 15 degrees of freedom are accommodated as follows

$$
\begin{gathered}
\left(\psi_{p}\right)_{L}=\left(\begin{array}{c}
d_{1}^{C} \\
d_{2}^{C} \\
d_{3}^{C} \\
e \\
\nu
\end{array}\right)_{L} \\
\chi_{L}^{p q}=\frac{1}{\sqrt{2}}\left(\begin{array}{ccccc}
0 & u_{3}^{C} & -u_{2}^{C} & -u_{1} & -d_{1} \\
-u_{3}^{C} & 0 & u_{1}^{C} & -u_{2} & -d_{2} \\
u_{2}^{C} & -u_{1}^{C} & 0 & -u_{3} & -d_{3} \\
u_{1} & u_{2} & u_{3} & 0 & -e^{C} \\
d_{1} & d_{2} & d_{3} & e^{C} & 0
\end{array}\right)_{L}
\end{gathered}
$$

and the electron and quark masses are

$$
m_{e}=m_{d}=\frac{g_{1} v_{\mathrm{EW}}}{\sqrt{2}} \quad m_{u}=4 g_{2} v_{\mathrm{EW}} .
$$

\section{SCATTERING FERMIONS OFF THE WALL}

We now use the equations of motion Eq. 600 and Eq. (61) to determine how fermions scatter off the $q=2$ domain wall. It is straightforward to see that the equations for $\left(\psi_{5,3}\right)_{L}$ and $\left(\chi_{35}\right)_{L}$ (that is, $\nu_{L}$ and $\left.d_{3}\right)$ are coupled, but decoupled from the other fermionic components. Hence initially we focus on these. Eq. 611 with $(p, q)=(3,5)$ yields

$$
i \not \partial \chi_{L}^{35}-\frac{m_{d}}{\sqrt{2}} \gamma^{0} C\left(\psi_{3}\right)_{L}^{*}=0
$$

while Eq. 601 with $p=5$ and $p=3$ gives, respectively

$$
\begin{gathered}
i \not \partial\left(\psi_{5}\right)_{L}+e \gamma^{2}\left(\boldsymbol{A}_{2}^{*}\right)_{53}\left(\psi_{3}\right)_{L}=0, \\
i \not \partial\left(\psi_{3}\right)_{L}+e \gamma^{2}\left(\boldsymbol{A}_{2}^{*}\right)_{35}\left(\psi_{5}\right)_{L}-\sqrt{2} m_{d} \gamma^{0} C\left(\chi_{L}^{35}\right)^{*}=0 .
\end{gathered}
$$

In the limit of a zero thickness wall, the gauge field is a $\delta$-function centered on the wall (see Eq. (58)), and Eqs. (66) and (67) give the matching conditions across the wall. Using [55), we obtain

$$
\begin{aligned}
& \frac{d\left(\psi_{5}\right)_{L}}{d \theta}+\left(\psi_{3}\right)_{L}=0 \\
& \frac{d\left(\psi_{3}\right)_{L}}{d \theta}-\left(\psi_{5}\right)_{L}=0
\end{aligned}
$$


so that on integrating and imposing the boundary conditions Eq. (52) we find

$$
\begin{aligned}
& \left.\left(\psi_{3}\right)_{L}\right|_{0^{-}}=\left.\left(\psi_{5}\right)_{L}\right|_{0^{+}} \\
& \left.\left(\psi_{5}\right)_{L}\right|_{0^{-}}=-\left.\left(\psi_{3}\right)_{L}\right|_{0^{+}}
\end{aligned}
$$

From Eq. (65), $\chi_{L}^{35}$ is continuous across the wall. Suppose a neutrino is incident on the wall from $-\infty,\left.\left(\psi_{5}\right)_{L}\right|_{0^{-}} \neq$ 0. Then from Eq. (69),$\left.\left(\psi_{3}\right)_{L}\right|_{0^{+}} \neq 0$ so that one expects a transmitted down quark. It is straightforward to solve this scattering problem. In the Dirac representation and using the projector $\left(1-\gamma^{5}\right) / 2$, the incident left-handed neutrino is given by (see Eq. [66)

$$
\left(\psi_{5}\right)_{L}^{i}(y<0)=e^{-i \omega(t-y)}\left(\begin{array}{c}
1 \\
-i \\
-1 \\
i
\end{array}\right)
$$

while the reflected neutrino and the different left handed components of the down quark are given by

$$
\begin{gathered}
\left(\psi_{5}\right)_{L}^{r}(y<0)=\alpha_{r} e^{-i \omega(t+y)}\left(\begin{array}{c}
1 \\
i \\
-1 \\
-i
\end{array}\right) \\
\left(\psi_{3}\right)_{L}^{r}(y<0)=e^{-i\left(\omega t+k^{\prime} y\right)}\left(\begin{array}{c}
p \\
q \\
-p \\
-q
\end{array}\right) \\
\left(\chi_{L}^{35}\right)^{r *}(y<0)=\frac{1}{\sqrt{2}} e^{-i\left(\omega t+k^{\prime} y\right)}\left(\begin{array}{c}
r \\
s \\
-r \\
-s
\end{array}\right)
\end{gathered}
$$

where, from Eqs. 65] and [67), $\omega^{2}-k^{2}=m_{d}^{2}$ and

$$
s=\frac{1}{m_{d}}\left(\omega p+i k^{\prime} q\right), r=-\frac{1}{m_{d}}\left(\omega q-i k^{\prime} p\right) .
$$

For $y>0$, the transmitted waves are

$$
\begin{aligned}
\left(\psi_{5}^{L}\right)_{t}(y>0) & =\alpha_{t} e^{-i \omega(t-y)}\left(\begin{array}{c}
1 \\
-i \\
-1 \\
i
\end{array}\right) \\
\left(\psi_{3}^{L}\right)_{t}(y>0) & =e^{-i\left(\omega t-k^{\prime} y\right)}\left(\begin{array}{c}
f \\
g \\
-f \\
-g
\end{array}\right) \\
\left(\chi_{L}^{35}\right)^{t *}(y>0) & =\frac{1}{\sqrt{2}} e^{-i\left(\omega t-k^{\prime} y\right)}\left(\begin{array}{c}
a \\
b \\
-a \\
-b
\end{array}\right)
\end{aligned}
$$

where

$$
b=\frac{1}{m_{d}}\left(\omega f-i k^{\prime} g\right), a=-\frac{1}{m_{d}}\left(\omega g+i k^{\prime} f\right) .
$$

From the boundary conditions Eqs. (68) and (69) we find

$$
\begin{gathered}
\alpha_{r}=0, \quad \alpha_{t}=\frac{\left(\omega-k^{\prime}\right)}{\omega+k^{\prime}}=p=i q \\
f=-1=i g \\
r=-i \frac{\left(\omega-k^{\prime}\right)}{m_{d}}=i s=a=i b .
\end{gathered}
$$

Since $\alpha_{r}=0$ there is no reflected neutrino. This could have been expected as a consequence of helicity conservation and the fact that the interaction of the domain wall does not depend on the spin orientation of the fermion. The incoming neutrino scatters either by reflection as a down quark, or gets transmitted as a neutrino or a down quark. Such lepton (and baryon) non-conserving processes have been studied in a number of other situations such as fermion scattering off $S U(5)$ monopoles [15, 16, 17]. Furthermore, notice that all the transmitted and reflected waves are proportional to the same spinor $\xi \equiv(1,-i,-1, i)^{\mathrm{T}}$, which is a left-handed eigenstate of the massless Dirac equation. The (massive) down quark spinors are also proportional to $\xi$, reflecting the fact that they are not momentum eigenstates - translational symmetry is broken by the presence of the wall.

This scattering process does not conserve electric charge: the incident electric current vanishes, while the transmitted and reflected electric currents are not equal and opposite. We find

$$
j_{r}=-\frac{\left(\omega-k^{\prime}\right)}{\left(\omega+k^{\prime}\right)} j_{t}
$$

The scattering must therefore be accompanied by the transfer of charge to the domain wall, and in a similar manner by a transfer of color charge. These different charges will be carried by the massless gauge excitations $\boldsymbol{A}_{y}$ living on the wall.

Here we have focused on an incoming $\nu_{L}$ scattering into $d_{3}$. These results can straightforwardly be generalized to both incoming neutrinos and $d_{3}$ 's, as well as the interactions between other particles (such as $d_{1}$ and $u_{2}^{C}$ see Eq. (61)).

\section{CONCLUSIONS}

In this paper we have studied a number of different aspects related to the domain walls formed in the spontaneous symmetry breaking of $S U(5) \times Z_{2} \rightarrow S U(3) \times$ $S U(2) \times U(1) / Z_{6}$, and in particular the effect of the subsequent symmetry breaking down to $S U(3) \times U(1) / Z_{3}$. As we have discussed, even before the second symmetry breaking, the presence of a second scalar field (the vector Higgs $\vec{\phi}$ ) can lead to the existence of a scalar condensate on the wall $\left(\phi_{1}\right)$ which is charged under $S U(3)$ and $U(1)$. The interaction of this condensate with magnetic 
monopoles also carrying $S U(3)$ and $U(1)$ charges can be diverse. For example, a monopole can either be repelled from the wall through a (generalized) Meissner effect; or it can be attracted to the wall with its flux threading the wall through a vortex configuration of $\phi_{1}$ on the wall. We aim to study these phenomena, and the seeming existence of massive fermions localized on the wall, in more detail in the future.

After the second symmetry breaking phase transition (the electroweak phase transition) when $\langle\vec{\phi}\rangle \neq 0$, fermions off the wall become massive. When a fermion scatters with the wall, lepton and baryon numbers can change, and we have shown explicitly that a neutrino can either scatter by reflection into a down quark, or be transmitted together with a down quark.

The $S U(5)$ grand unified model we have used for motivation for this work is not phenomenologically viable, and similarly a network of domain walls is not cosmolog- ically viable (though this second issue can be resolved by adding a small $\operatorname{Tr} \boldsymbol{H}^{3}$ term to the model we have studied: the domain walls still exist but they now eventually decay). Despite these comments we believe that our work is of interest as it illustrates some of the very complex properties of grand unified models which have not been fully probed in the past. Furthermore the effects we have highlighted here may appear in other models or indeed in other systems.

\section{Acknowledgments}

We are grateful to Levon Pogosian for collaboration in the initial stages of this project. This work was supported by the DOE and NASA at Case, and by the University of Paris VII.
[1] M. Trodden, "Electroweak baryogenesis," Rev. Mod. Phys. 71 (1999) 1463 arXiv:hep-ph/9803479.

[2] A. Vilenkin and E.P.S. Shellard, "Cosmic strings and other topological defects", Cambridge University Press (1994).

[3] R. H. Brandenberger, W. Kelly and M. Yamaguchi, "Electroweak baryogenesis with embedded domain walls," arXiv:hep-ph/0503211.

[4] L. Pogosian and T. Vachaspati, "Domain walls in SU(5)," Phys. Rev. D 62, 123506 (2000)

[5] T. Vachaspati, "A class of kinks in SU(N) $\times \mathrm{Z}(2)$," Phys. Rev. D 63 (2001) 105010 arXiv:hep-th/0102047.

[6] L. Pogosian and T. Vachaspati, "Space of kink solutions in SU(N) × Z(2)," Phys. Rev. D 64 (2001) 105023 arXiv:hep-th/0105128.

[7] T. Vachaspati, "Symmetries within domain walls," Phys. Rev. D 67 (2003) 125002 arXiv:hep-th/0303137.

[8] L. Pogosian and T. Vachaspati, "Domain wall lattices," Phys. Rev. D 67 (2003) 065012 arXiv:hep-th/0210232.

[9] N. D. Antunes, L. Pogosian and T. Vachaspati, "On formation of domain wall lattices," Phys. Rev. D 69 (2004) 043513 arXiv:hep-ph/0307349.
[10] N. D. Antunes and T. Vachaspati, "Spontaneous formation of domain wall lattices in two spatial dimensions," Phys. Rev. D 70 (2004) 063516 arXiv:hep-ph/0404227.

[11] D. Bailin and A. Love, "Introduction to Gauge Field Theory Edition", Institute of Physics Publishing (1993).

[12] T. Cheng and L. Li, "Gauge Theory of Elementary Particle Physics", Oxford University Press (1984).

[13] A. Ayala, J. Jalilian-Marian, L. D. McLerran and A. P. Vischer, "Scattering in the presence of electroweak phase transition bubble walls," Phys. Rev. D 49 (1994) 5559 arXiv:hep-ph/9311296.

[14] G. R. Farrar and J. W. McIntosh, "Scattering from a domain wall in a spontaneously broken gauge theory," Phys. Rev. D 51, 5889 (1995) arXiv:hep-ph/9412270.

[15] C. P. Dokos and T. N. Tomaras, "Monopoles And Dyons In The SU(5) Model," Phys. Rev. D 21 (1980) 2940.

[16] V. A. Rubakov, "Adler-Bell-Jackiw Anomaly And Fermion Number Breaking In The Presence Of A Magnetic Monopole," Nucl. Phys. B 203 (1982) 311.

[17] C. G. Callan, "Dyon - Fermion Dynamics," Phys. Rev. D 26 (1982) 2058. 